\title{
Impact of Left Ventricular Afterload on Longitudinal Dyssynchrony in Patients With Severe Aortic Stenosis and Preserved Ejection Fraction
}

Kohei Yamawaki, MD; Hidekazu Tanaka, MD, PhD; Kensuke Matsumoto, MD;

Mana Hiraishi, MD; Tatsuya Miyoshi, MD; Akihiro Kaneko, MD; Takayuki Tsuji, MD;

Keiko Ryo, MD; Kazuko Norisada, MD, PhD; Yuko Fukuda, MD; Kazuhiro Tatsumi, MD, PhD;

Tetsuari Onishi, MD, PhD; Kenji Okada, MD, PhD; Yutaka Okita, MD, PhD;

Hiroya Kawai, MD, PhD; Ken-ichi Hirata, MD, PhD

Background: The purpose of this study was to investigate whether patients with severe aortic stenosis (AS) and preserved ejection fraction (EF) have dyssynchrony and whether it improves after aortic valve replacement (AVR).

Methods and Results: We studied 30 consecutive patients with severe AS and preserved EF undergoing AVR. For baseline comparison, we studied 17 EF-matched patients with mild-to-moderate AS, and 18 EF-matched normal volunteers. Longitudinal dyssynchrony was determined as the standard deviation for time-to-peak speckle-tracking strain in apical 4- and 2-chamber views at the basal- and mid-levels. Radial and circumferential dyssynchrony was determined as the difference for time-to-peak strain between the anteroseptum and posterior wall from the mid-left ventricular (LV) short-axis view. Each of the myocardial functions was also evaluated by averaging each peak systolic strain. Longitudinal dyssynchrony and function in patients with severe AS was significantly worse than in the patients with mild-to-moderate AS and the controls ( $94 \pm 46$ vs. $66 \pm 18 \mathrm{~ms}^{*}$ and $52 \pm 17 \mathrm{~ms}^{*}$, and $12.5 \pm 3.7 \%$ vs. $16 \pm 3.5 \%{ }^{*}$ and $18.7 \pm 3.7 \%{ }^{*}$, respectively, ${ }^{*} \mathrm{P}<0.05$, vs. severe AS). In contrast, radial and circumferential dyssynchrony were similar for the 3 groups. Importantly, the dyssynchrony of patients with severe AS significantly improved after AVR from $94 \pm 46 \mathrm{~ms}$ to $68 \pm 22 \mathrm{~ms}(\mathrm{P}<0.005)$.

Conclusions: Significant longitudinal dyssynchrony was present in patients with severe AS and preserved EF, and it improved after AVR. (Circ J 2012; 76: 744-751)

Key Words: Asynchrony; Echocardiography; Myocardial contraction; Valvular disease

A ortic stenosis (AS) is a common native valvular heart disease, and aortic valve replacement (AVR) is the only effective treatment that can halt the natural course of progression for such patients. ${ }^{1,2}$ AVR is indicated when ejection fraction (EF) is reduced or when symptoms are revealed during exercise testing based on the current guidelines. ${ }^{3,4}$ Although EF is the most widely used measurement of left ventricular (LV) function upon which surgical risk assessment is based, it is reduced only in patients with end-stage AS. Currently, the management of patients with severe AS and preserved EF remains controversial..$^{5-7}$ Previous investigators have demonstrated that the fibrotic changes induced by AS start in the subendocardium and affect mainly longitudi- nal myocardial function, which is not clearly reflected by the EF. ${ }^{8-14}$ Thus, the occurrence of reduced longitudinal myocardial function may affect the clinical outcome for patients with preserved EF.

LV dyssynchrony has emerged as an important mechanism contributing to the progression of heart failure (HF) and ventricular remodeling, and appears to play a pathophysiologic role in HF. Dyssynchrony has been typically studied in HF patients with wide QRS duration, but the relationship between LV dyssynchrony and AS has not been clarified.

Accordingly, the purpose of this study was to investigate, by means of multidirectional assessment of 2-dimensional (2D) speckle-tracking strain whether patients with AS and pre-

Received September 27, 2011; revised manuscript received November 2, 2011; accepted November 7, 2011; released online December 28, 2011 Time for primary review: 6 days

Division of Cardiovascular Medicine, Department of Internal Medicine (K.Y., H.T., K.M., M.H., T.M., A.K., T.T., K.R., K.N., Y.F., K.T., T.O., H.K., K.H.), Division of Cardiovascular Surgery, Department of Surgery (K.O., Y.O.), Kobe University Graduate School of Medicine, Kobe, Japan

Mailing address: Hidekazu Tanaka, MD, PhD, Division of Cardiovascular Medicine, Department of Internal Medicine, Kobe University Graduate School of Medicine, 7-5-1 Kusunoki-cho, Chuo-ku, Kobe 650-0017, Japan. E-mail: tanakah@med.kobe-u.ac.jp

ISSN-1346-9843 doi:10.1253/circj.CJ-11-1098

All rights are reserved to the Japanese Circulation Society. For permissions, please e-mail: cj@j-circ.or.jp 


\begin{tabular}{|c|c|c|c|}
\hline & $\begin{array}{c}\text { Severe AS } \\
(n=30)\end{array}$ & $\begin{array}{l}\text { Mild-to-moderate AS } \\
(\mathrm{n}=17)\end{array}$ & $\begin{array}{l}\text { Normal control } \\
\quad(n=18)\end{array}$ \\
\hline Age (years) & $73 \pm 7$ & $75 \pm 8$ & $70 \pm 10$ \\
\hline$M / F$ & $10 / 20$ & $7 / 10$ & $9 / 9$ \\
\hline Body surface area $\left(\mathrm{m}^{2}\right)$ & $1.51 \pm 0.17$ & $1.50 \pm 0.18$ & $1.58 \pm 0.17$ \\
\hline Systolic blood pressure (mmHg) & $119 \pm 16$ & $123 \pm 14$ & $114 \pm 15$ \\
\hline \multicolumn{4}{|l|}{ Etiology of aortic stenosis } \\
\hline Congenital & 11 & 2 & \\
\hline Rheumatic & 0 & 0 & \\
\hline Degenerative & 19 & 15 & \\
\hline LV end-diastolic diameter (mm) & $45 \pm 7$ & $44 \pm 5$ & $44 \pm 4$ \\
\hline LV end-systolic diameter (mm) & $29 \pm 9^{\dagger}$ & $26 \pm 4$ & $27 \pm 3$ \\
\hline LV mass index $\left(\mathrm{g} / \mathrm{m}^{2}\right)$ & $171 \pm 43^{*}+$ & $139 \pm 37^{*}$ & $89 \pm 16$ \\
\hline LV wall stress (kdyn/cm²) & $69 \pm 33^{*}, \dagger$ & $45 \pm 14$ & $46 \pm 13$ \\
\hline Global LV afterload $\left(\mathrm{mmHg} \cdot \mathrm{m}^{-2} \cdot \mathrm{ml}^{-1}\right)$ & $3.6 \pm 0.6^{\star, \dagger}$ & $2.8 \pm 0.7$ & $2.8 \pm 0.6$ \\
\hline LV end-diastolic volume (ml) & $74 \pm 30^{*}$ & $65 \pm 16$ & $60 \pm 16$ \\
\hline LV end-systolic volume (ml) & $30 \pm 24^{*}, \dagger$ & $21 \pm 6$ & $20 \pm 7$ \\
\hline LV ejection fraction (\%) & $63 \pm 14$ & $67 \pm 7$ & $67 \pm 6$ \\
\hline AVA mean gradient $(\mathrm{mmHg})$ & $61 \pm 27^{*, \dagger}$ & $23 \pm 11^{*}$ & $4 \pm 2$ \\
\hline $\operatorname{AVA}\left(\mathrm{cm}^{2}\right)$ & $0.70 \pm 0.18^{\star}, \dagger$ & $1.23 \pm 0.20^{*}$ & $2.67 \pm 0.44$ \\
\hline
\end{tabular}

Data are expressed as mean \pm SD or number.

${ }^{*} \mathrm{P}<0.05$ vs. normal control, ${ }^{\mathrm{T}} \mathrm{P}<0.05$ vs. mild-to-moderate $A S$.

AS, aortic stenosis; LV, left ventricular; AVA, aortic valve area.

served EF have LV dyssynchrony and whether it improves after AVR.

\section{Methods}

\section{Study Population}

The study group consisted of 33 consecutive patients with severe AS and preserved EF, defined as aortic valve area (AVA) $<1.0 \mathrm{~cm}^{2}$, who underwent AVR; 3 patients (9\%) with suboptimal echocardiographic images were excluded from all subsequent analyses. The eventual patient study group thus consisted of 30 patients with severe AS (Table 1), 20 (67\%) of whom were female. The group's mean age was $73 \pm 7$ years, EF was $63 \pm 14 \%$ (all $\geq 50 \%$ ), and QRS duration was $99 \pm 21 \mathrm{~ms}$ (all $<120 \mathrm{~ms}$ ). For baseline comparison, we also studied 17 ageand EF-matched asymptomatic patients with mild-to-moderate AS (AVA: $1.0-1.6 \mathrm{~cm}^{2}$; mean age: $75 \pm 8$ years; EF: $67 \pm 7 \%$ ), and 18 age- and EF-matched normal volunteers (mean age: $70 \pm 10$ years; EF: $67 \pm 6 \%$ ). We excluded AS patients with: (1) coronary artery disease, defined as visually 1- or multivessel coronary artery stenosis of a major epicardial vessel $>50 \%$ or a previous history of myocardial infarction; (2) atrial fibrillation; (3) $\mathrm{EF}<50 \%$; (4) more than mild additional valve disease; and (5) presence of regional wall motion abnormality. The normal volunteers had no history of cardiovascular disease and completely normal electrocardiograms as well as 2D and Doppler echocardiograms. Written informed consent was given by all patients.

\section{Echocardiography}

All echocardiographic data were acquired with a commercially available echocardiography system (Vivid 7; GE-Vingmed Ultrasound AS, Horten, Norway).

Digital routine grayscale 2D cine loops from 3 consecutive beats were obtained at end-expiratory apnea from standard apical views (4-chamber, 2-chamber, and long-axis), parasternal long-axis view, and mid-LV short-axis views at depths of
$11-20 \mathrm{~cm}$ (mean $16 \pm 2 \mathrm{~cm}$ ). Frame rates were $44-90 \mathrm{~Hz}$ (mean $59 \pm 11 \mathrm{~Hz}$ ) for grayscale imaging. Sector width was optimized to allow for complete myocardial visualization while maximizing frame rate.

LV dimensions were calculated from the standard M-mode images obtained in the parasternal long-axis views and included LV diameters and end-diastolic thicknesses of the interventricular septum and posterior wall. LV mass was calculated using the formula proposed by Devereux et al and corrected by the body surface area to derive LV mass index. ${ }^{15}$ The LV volumes and EF were assessed by biplane Simpson's rule using manual tracing of digital images. ${ }^{16}$ For global LV afterload, the valvulo-arterial impedance was calculated using the formula presented by Briand et al. ${ }^{17}$ End-systolic wall stress, as a measure of LV afterload, was also calculated using the formula introduced by Reichek et al. ${ }^{18}$ Follow-up echocardiograms were available for 30 patients with severe AS $13 \pm 7$ days after AVR.

\section{Definition of AS}

Definition of aortic sclerosis was based on recommendations by the European Association of Echocardiography and American Society of Echocardiography. ${ }^{4}$ AS etiologies were defined as congenital, rheumatic, or degenerative as previously published.,19 Classification of AS severity was based on AVA peak velocity and mean gradient. AVA was calculated with the continuity equation using velocity-time integrals of the aorta and LV outflow tract. Peak and mean aortic transvalvular gradients were calculated using the modified Bernoulli equation. ${ }^{3}$

\section{Speckle-Tracking Strain Analysis}

Speckle-tracking strain analysis was performed offline using dedicated software (EchoPAC version BTO8; GE-Vingmed Ultrasound AS) to assess LV dyssynchrony and myocardial function. LV dyssynchrony was assessed for each patient by longitudinal, radial and circumferential speckle-tracking strain (Figure 1). Longitudinal dyssynchrony was evaluated as the 


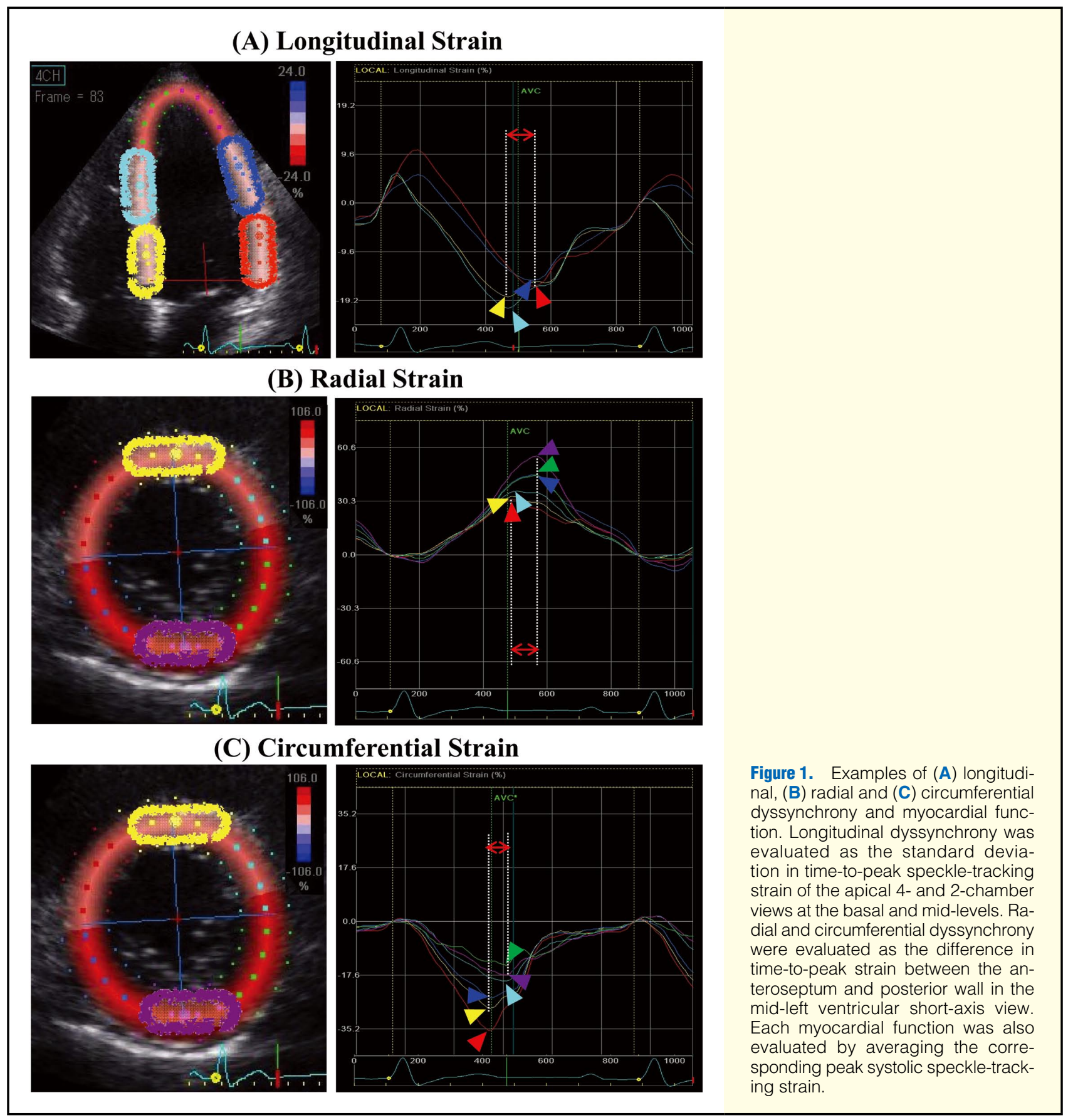

standard deviation in time-to-peak speckle-tracking strain in the apical 4- and 2-chamber views at the basal- and mid-levels. Both radial and circumferential dyssynchrony were evaluated as the difference in the time-to-peak strain between the anteroseptum and posterior wall using the mid-LV short-axis view. ${ }^{20}$ Longitudinal, radial and circumferential myocardial functions were also evaluated by averaging each of the corresponding peak systolic speckle-tracking strains (Figure 1).

\section{Statistical Analysis}

All group data were compared by 2-tailed Student's t-test for paired and unpaired data and are presented as mean \pm SD. Proportional differences were evaluated by Fisher's exact test or the $X^{2}$ test as appropriate. Correlation analysis was performed with linear regression, and the results are expressed as Pearson's correlation coefficients. Differences in baseline characteristics between groups were assessed by 1- or 2-way analysis of variance followed by a Tukey multiple comparisons test. Univariate linear regression analysis was initially used to assess the relationship between longitudinal dyssynchrony and echocardiographic parameters. Multiple linear regression analysis based on stepwise selection was then performed for an analysis of independent determinants of longitudinal dyssynchrony. The entry criterion for the multivariate linear regression model was a univariate $\mathrm{P}$ value $<0.05$. Inter- and intra-observer variabilities were expressed as the absolute difference between the respective measurements divided by their mean value from 10 randomly selected patients. For all 


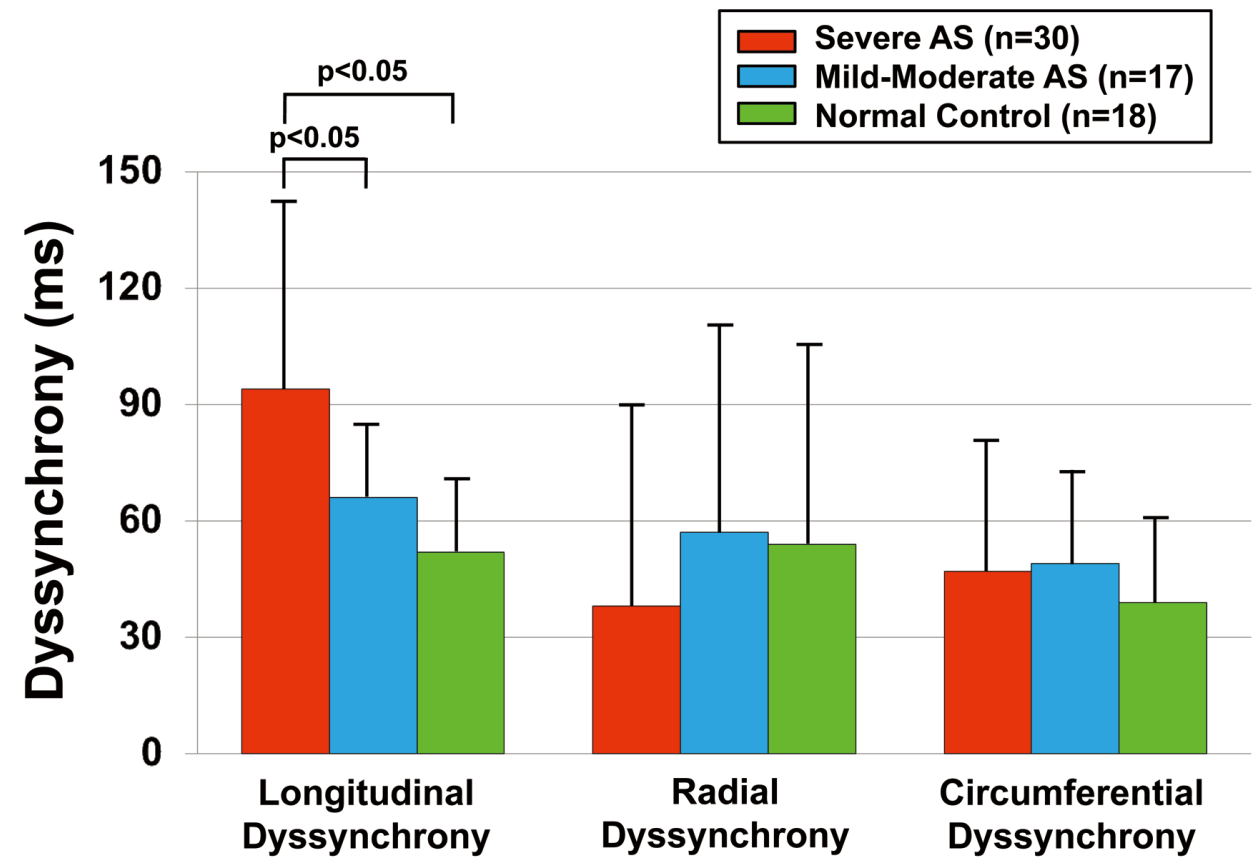

Figure 2. Comparison of baseline left ventricular dyssynchrony among the 3 groups, demonstrating that longitudinal dyssynchrony in patients with severe aortic stenosis (AS) was significantly greater than in patients with mild-to-moderate AS or the normal controls, but radial and circumferential dyssynchrony were similar for the groups.

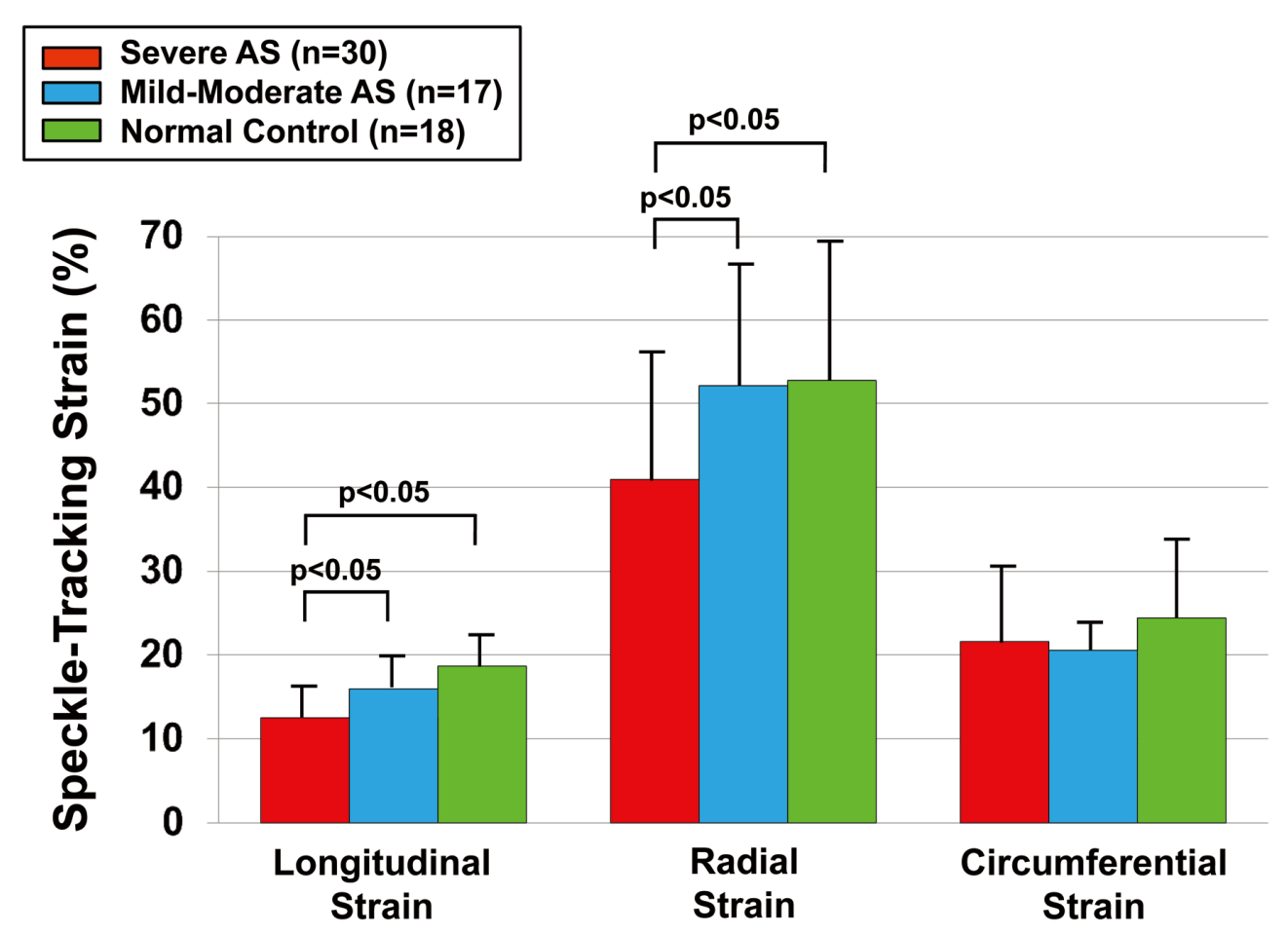

Figure 3. Comparison of baseline left ventricular myocardial function among the 3 groups, demonstrating that both longitudinal and radial strain in patients with severe aortic stenosis (AS) were significantly lower than in patients with mild-to-moderate AS or the normal controls. In contrast, circumferential strain was similar in all groups. 


\begin{tabular}{|c|c|c|c|}
\hline & Before AVR & After AVR & $P$ value \\
\hline Systolic blood pressure (mmHg) & $119 \pm 16$ & $113 \pm 13$ & $<0.05$ \\
\hline LV end-diastolic diameter (mm) & $45 \pm 7$ & $41 \pm 7$ & $<0.05$ \\
\hline LV end-systolic diameter (mm) & $29 \pm 9$ & $26 \pm 7$ & $<0.05$ \\
\hline LV mass index $\left(\mathrm{g} / \mathrm{m}^{2}\right)$ & $171 \pm 43$ & $160 \pm 40$ & $<0.05$ \\
\hline LV wall stress $\left(\mathrm{kdyn} / \mathrm{cm}^{2}\right)$ & $69 \pm 33$ & $39 \pm 18$ & $<0.05$ \\
\hline Global LV afterload $\left(\mathrm{mmHg} \cdot \mathrm{m}^{-2} \cdot \mathrm{ml}^{-1}\right)$ & $3.6 \pm 0.6$ & $3.0 \pm 0.6$ & $<0.05$ \\
\hline LV end-diastolic volume (ml) & $74 \pm 30$ & $59 \pm 22$ & $<0.05$ \\
\hline LV end-systolic volume (ml) & $30 \pm 24$ & $23 \pm 15$ & $<0.05$ \\
\hline LV ejection fraction (\%) & $63 \pm 14$ & $63 \pm 9$ & NS \\
\hline AVA mean gradient $(\mathrm{mmHg})$ & $61 \pm 27$ & $17 \pm 6$ & $<0.05$ \\
\hline $\operatorname{AVA}\left(\mathrm{cm}^{2}\right)$ & $0.70 \pm 0.18$ & $1.42 \pm 0.32$ & $<0.05$ \\
\hline
\end{tabular}

Data are expressed as mean \pm SD or number.

AVR, aortic valve replacement; NS, not significant. Other abbreviations see in Table 1.

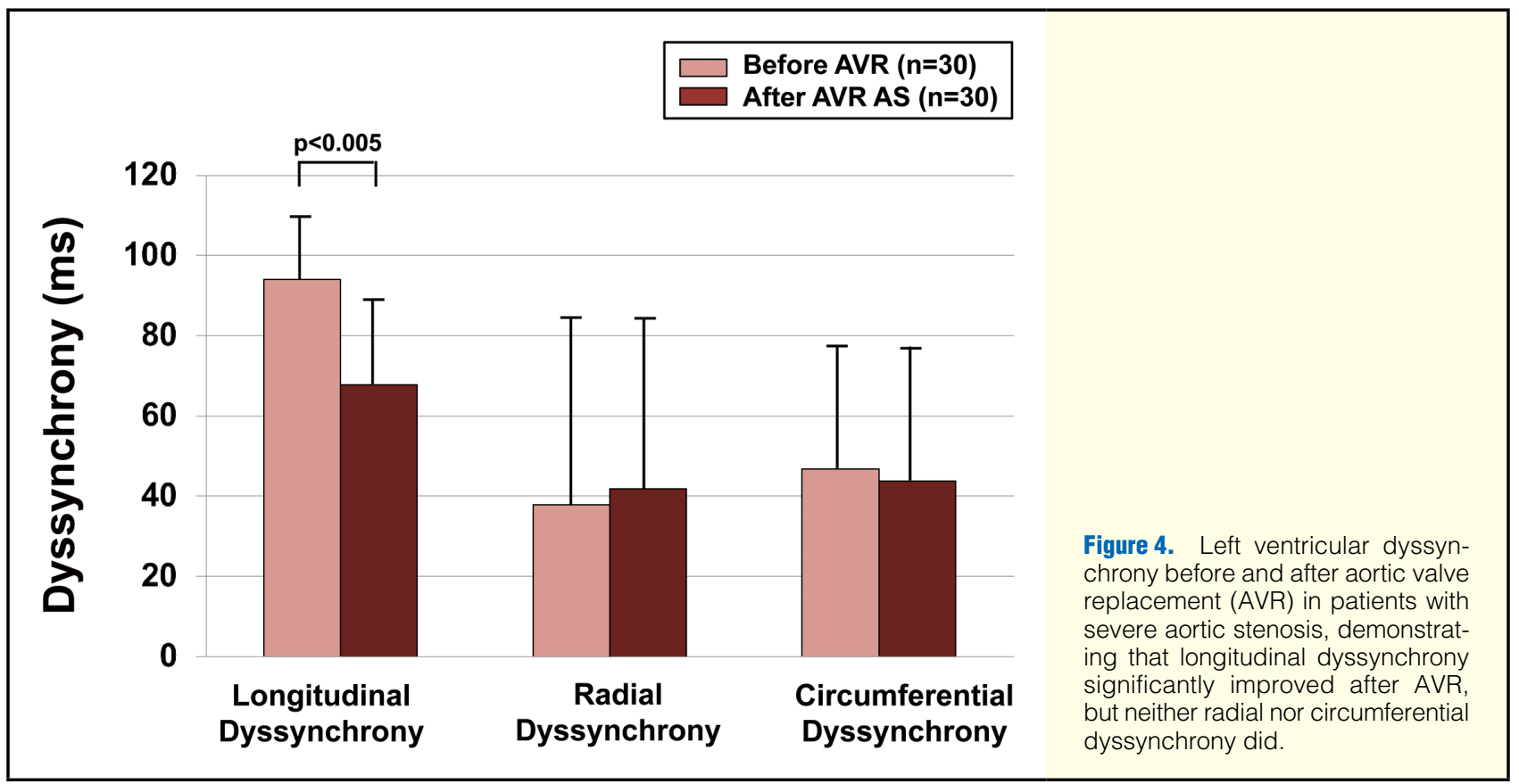

tests, $\mathrm{P}<0.05$ was considered statistically significant. All the analyses were performed with software $\mathrm{R}$ version 2.11.1. ( $\mathrm{R}$ foundation for Statistical Computing, Vienna, Austria). The authors had full access to the data and take full responsibility for its integrity. All authors have read and agree to the manuscript as written.

\section{Results}

The baseline clinical and echocardiographic characteristics of the 65 subjects are summarized in Table 1. Overall, speckletracking analysis was possible for $96 \%$ of 1,520 attempted segments from 95 subjects (baseline for 65 subjects and follow-up for 30 patients with severe AS) with technically satisfactory images. Only $4 \%$ of the segments had to be eliminated. The inter- and intra-observer variabilities were $11 \pm 9 \%$ and $10 \pm 6 \%, 9 \pm 9 \%$ and $10 \pm 7 \%$, and $12 \pm 8 \%$ and $11 \pm 5 \%$ for the analysis of longitudinal, radial and circumferential dyssynchrony, respectively.

\section{Baseline LV Dyssynchrony and Myocardial Function in Patients With AS}

Longitudinal dyssynchrony of patients with severe AS was significantly greater than that of patients with mild-to-moderate $\mathrm{AS}$ or the controls $(94 \pm 46 \mathrm{vs} .66 \pm 18 \mathrm{~ms} *$ and $52 \pm 17 \mathrm{~ms} *$, respectively; ${ }^{*} \mathrm{P}<0.05$, vs. severe AS, Figure 2$)$. On the other hand, both the radial and circumferential dyssynchrony of the 3 groups were similar ( $38 \pm 49$ vs. $58 \pm 51 \mathrm{~ms}$ and $54 \pm 48 \mathrm{~ms}$, and $47 \pm 32$ vs. $49 \pm 23 \mathrm{~ms}$ and $39 \pm 21 \mathrm{~ms}$, Figure 2). Longitudinal and radial strains for patients with severe AS were significantly lower than those for patients with mild-to-moderate AS and the controls $(12.5 \pm 3.7 \%$ vs. $16 \pm 3.5 \% *$ and $18.7 \pm$ $3.7 \% *, 40.9 \pm 15.7 \%$ vs. $52.2 \pm 14.6 \% *$ and $52.8 \pm 16.8 \% *$, respectively, ${ }^{*} \mathrm{P}<0.05$, vs. severe AS, Figure 3 ). In contrast, the circumferential strain of the 3 groups was similar $(21.6 \pm 9.4 \%$ vs. $20.6 \pm 3.1 \%$ and $24.4 \pm 9.9 \%$, Figure 3 ).

In addition, longitudinal dyssynchrony was significantly correlated with mitral inflow E and mitral E' annular velocity ratio $(r=0.45$ and $\mathrm{P}=0.01)$, but not with brain natriuretic pep- 


\begin{tabular}{|c|c|c|c|c|c|c|}
\hline & \multicolumn{6}{|c|}{ Longitudinal dyssynchrony (dependent variable) } \\
\hline & \multicolumn{3}{|c|}{ Univariate analysis } & \multicolumn{3}{|c|}{ Multivariate analysis } \\
\hline & $\beta$ coefficient & $95 \% \mathrm{Cl}$ & $P$ value & $\beta$ coefficient & $95 \% \mathrm{Cl}$ & $P$ value \\
\hline LV mass index $\left(\mathrm{g} / \mathrm{m}^{2}\right)$ & 0.143 & -0.044 to 0.2500 & 0.168 & & & \\
\hline LV wall stress (kdyn/cm²) & -0.123 & -0.435 to 1.109 & 0.236 & & & \\
\hline Global LV afterload $\left(\mathrm{mmHg} \cdot \mathrm{m}^{-2} \cdot \mathrm{ml}^{-1}\right)$ & 0.222 & 0.997 to 20.322 & 0.031 & 0.102 & -5.213 to 15.002 & 0.339 \\
\hline LV end-systolic volume (ml) & -0.146 & -0.724 to 0.120 & 0.158 & & & \\
\hline LV ejection fraction (\%) & 0.150 & -0.185 to 1.221 & 0.147 & & & \\
\hline Longitudinal strain (\%) & 0.346 & 1.257 to 4.435 & 0.001 & 0.306 & 0.791 to 4.246 & 0.005 \\
\hline
\end{tabular}

Adjusted R-squared 0.129 .

$\mathrm{Cl}$, confidence interval. Other abbreviation see in Table 1.

tide levels ( $\mathrm{r}=-0.22$ and $\mathrm{P}=0.25)$. Using a dichotomized longitudinal dyssynchrony value (high or low), longitudinal dyssynchrony was not significantly associated with clinical characteristics including symptoms, NYHA functional class, blood pressure, or heart rate.

\section{Dyssynchrony After AVR of Patients With Severe AS}

Follow-up echocardiographic data were available for 30 patients with severe AS who underwent AVR (Table 2). Bioprostheses were implanted in all patients $(100 \%)$; none $(0 \%)$ received mechanical prostheses. AVA increased significantly after AVR from $0.70 \pm 0.18 \mathrm{~cm}^{2}$ to $1.42 \pm 0.32 \mathrm{~cm}^{2}(\mathrm{P}<0.001)$, but AVR did not affect $\mathrm{EF}$, which remained virtually unchanged from $63 \pm 14 \%$ to $63 \pm 9 \%$. Importantly, the longitudinal dyssynchrony of patients with severe AS improved significantly after AVR from $94 \pm 46 \mathrm{~ms}$ to $68 \pm 22 \mathrm{~ms}$ ( $\mathrm{P}<0.005$, Figure 4), whereas neither radial nor circumferential dyssynchrony changed significantly ( $38 \pm 49$ vs. $42 \pm 45 \mathrm{~ms}$ and $47 \pm 32$ vs. $44 \pm$ $35 \mathrm{~ms}$, respectively, Figure 4).

\section{Predictors of Longitudinal Dyssynchrony}

Univariate analysis showed that longitudinal strain $(\beta=0.346$, $\mathrm{P}=0.001)$ and global $\mathrm{LV}$ afterload $(\beta=0.222, \mathrm{P}=0.031)$ were associated with longitudinal dyssynchrony (Table 3 ). Multivariable analysis showed that only longitudinal strain $(\beta=0.306$, $\mathrm{P}=0.005$ ) was an independent determinant of longitudinal dyssynchrony (Table 3 ).

\section{Discussion}

In this study, significant longitudinal dyssynchrony was present in patients with severe AS and preserved EF, and it improved after AVR. Furthermore, the longitudinal dyssynchrony in these patients might be related to LV afterload and associated with impaired longitudinal myocardial function, and as the AS severity of AS increases, this impairment progresses. These findings suggest that non-uniformity of cardiomyocyte damage may lead to LV dyssynchrony. To the best of our knowledge, ours is the first study to assess and identify in patients the association of LV dyssynchrony with AS and preserved EF.

\section{Longitudinal Myocardial Dysfunction in Patients With AS} Previous studies have demonstrated impaired longitudinal myocardial function in patients with AS. ${ }^{8-14}$ Carasso et al used speckle-tracking strain to demonstrate that longitudinal function was reduced in patients with severe AS and preserved EF and that it normalized after AVR. ${ }^{8}$ Moreover, Lindqvist et al showed that longitudinal strain, assessed by means of tissue
Doppler and speckle-tracking techniques, in patients with AS and preserved EF was significantly reduced, compared with age- and sex-matched normal controls, and that it improved after AVR. ${ }^{13}$ In the same vein, $\mathrm{Ng}$ et al recently reported longitudinal, circumferential and radial speckle-tracking strain with an increase in AS severity in patients with AS and preserved EF. ${ }^{10}$ Furthermore, Delgado et at observed that impaired longitudinal, circumferential, and radial speckle-tracking strain showed significant improvement after AVR in patients with severe AS and preserved EF. ${ }^{12}$ Our study confirms the findings of these previous investigations; namely, that LV myocardial function, including longitudinal function, is significantly impaired in patients with severe AS and preserved EF. A decrease or increase in a particular regional strain must be compensated by an increase or decrease in another strain to keep the global LVEF normal, but in this study longitudinal and radial strains were significantly different and only circumferential strain was similar between the 3 groups, despite similar EFs. Although the precise reason is unknown, we speculate that some other regional myocardial functional parameters, such as LV torsion, play a role in maintaining LVEF. Thus, multidirectional myocardial analysis may well be important for a better understanding of subtle myocardial dysfunction in AS patients. Furthermore, no significant correlation was observed between longitudinal dyssynchrony and circumferential $(r=0.13$ and $\mathrm{P}=0.34)$, or radial strain $(\mathrm{r}=0.07$ and $\mathrm{P}=0.59)$.

LV hypertrophy compensates for pressure overload in patients with AS, and may be accompanied by interstitial myocardial fibrosis that starts at the subendocardium and progresses toward replacement fibrosis. These alterations gradually affect LV function, thus contributing to the symptoms. ${ }^{21,22}$ Furthermore, longitudinal function is governed by the subendocardial myocardial fibers, which are aligned longitudinally and more sensitive to microvascular ischemia. ${ }^{12,23}$ The selective impairment in longitudinal function in patients with AS is thus related to the increase in subendocardial stress and associated reduction in coronary flow reserve. Consequently, the improvement in coronary flow reserve after AVR, secondary to an increase in the effective orifice area, results in a more efficient myocardial arterial supply and improved longitudinal function.

\section{Longitudinal Dyssynchrony in Patients With AS}

LV dyssynchrony has emerged as an important mechanism contributing to the progression of $\mathrm{HF}$ and ventricular remodeling, and appears to play a pathophysiologic role in $\mathrm{HF}^{24,25}$ Furthermore, LV dyssynchrony has various detrimental effects on LV systolic and diastolic functions, as well as right ventricular and left atrial functions, and it is well known that 
speckle-tracking strain is useful for quantifying LV dyssynchrony. ${ }^{20,26}$ However, LV dyssynchrony has been typically studied in HF patients with wide QRS duration, and little is known about the relationship between LV dyssynchrony and valvular heart disease. We found significant longitudinal dyssynchrony in patients with severe AS and preserved EF, and that it improved after AVR. Therefore, longitudinal dyssynchrony might be related to LV afterload and associated with longitudinal myocardial function. As previously mentioned, some studies have demonstrated impaired longitudinal myocardial function in patients with AS. Accordingly, there is great interest in the time course of the deterioration of longitudinal myocardial function and dyssynchrony in patients with AS. In our study, we observed progressive impairment of longitudinal dyssynchrony with an increase in AS severity, and longitudinal dyssynchrony was significantly correlated with longitudinal speckle-tracking strain in all subjects $(\mathrm{r}=0.35$ and $\mathrm{P}=0.001$ ). Thus, longitudinal myocardial dysfunction may lead to longitudinal dyssynchrony in patients with AS.

Regional myocardial dysfunction in patients with severe $\mathrm{AS}$ and preserved EF was not uniform in this study. Because the LV is not spherical, the differences in wall stress within the LV may be exaggerated under the increased afterload in severe AS patients, and may be reversed after AVR, resulting in the changes in LV longitudinal dyssynchrony observed in this study. Alternatively, however, non-uniformity of cardiomyocyte damage may also play a role in LV dyssynchrony, although we had no data regarding cardiomyocyte damage in this study.

\section{Clinical Implications}

Although EF is the most widely used measurement of LV function upon which surgical risk assessment is based, it is reduced only in patients with end-stage AS. As already mentioned, fibrotic changes induced by AS start in the subendocardium and affect mainly longitudinal myocardial function, which is not well reflected by the EF. Our study provides evidence of progressive impairment of longitudinal dyssynchrony with an increase in AS severity. Therefore, early detection of longitudinal dyssynchrony, as well as longitudinal function, may facilitate early identification of patients at risk of irreversible myocardial damage. Furthermore, the combined assessment of longitudinal dyssynchrony and myocardial function by means of speckle-tracking strain can further enhance the ability of this method to identify subclinical dysfunction in patients with severe AS and preserved EF.

\section{Study Limitations}

This study covered a small number of patients in a single-center study, so future studies of larger patient populations are necessary to assess our findings. Furthermore, only acute follow-up data after AVR were available, and long-term followup data were not part of this study. Limitations of the speckletracking strain analyses include endocardial border tracing where care must be taken to manually fine-tune the region of interest before generating and measuring regional strain. Finally, we should have determined the radial, circumferential, and longitudinal speckle-tracking strains simultaneously because LV performance is actually a 3-dimensional (3D) phenomenon. A newly developed 3D speckle-tracking system appears to be able to make such simultaneous measurements, ${ }^{27,28}$ and it could be helpful for the assessment of progressive subendocardial to transmural impairment of myocardial function with an increase in AS severity and chronic pressure overload.

\section{Conclusions}

Significant longitudinal dyssynchrony was present in patients with severe AS and preserved EF, and it improved after AVR. Longitudinal dyssynchrony in patients with AS may be associated with LV afterload and impaired longitudinal myocardial function.

\section{References}

1. Hiraoka A, Kuinose M, Chikazawa G, Totsugawa T, Katayama K, Yoshitaka H. Minimally invasive aortic valve replacement surgery: Comparison of port-access and conventional standard approach. Circ J 2011; 75: 1656-1660.

2. Spaccarotella C, Mongiardo A, Indolfi C. Pathophysiology of aortic stenosis and approach to treatment with percutaneous valve implantation. Circ J 2010; 75: 11-19.

3. Bonow RO, Carabello BA, Kanu C, de Leon AC Jr, Faxon DP, Freed $\mathrm{MD}$, et al. Acc/aha 2006 guidelines for the management of patients with valvular heart disease: A report of the american college of cardiology/american heart association task force on practice guidelines (writing committee to revise the 1998 guidelines for the management of patients with valvular heart disease): Developed in collaboration with the society of cardiovascular anesthesiologists: Endorsed by the society for cardiovascular angiography and interventions and the society of thoracic surgeons. Circulation 2006; 114: e84-e231.

4. Baumgartner H, Hung J, Bermejo J, Chambers JB, Evangelista A, Griffin BP, et al. Echocardiographic assessment of valve stenosis: EAE/ASE recommendations for clinical practice. J Am Soc Echocardiogr 2009; 22: 1-23; quiz 101-102.

5. Vahanian A, Otto CM. Risk stratification of patients with aortic stenosis. Eur Heart J 2010; 31: 416-423.

6. Baumgartner H, Otto CM. Aortic stenosis severity: Do we need a new concept? J Am Coll Cardiol 2009; 54: 1012-1013.

7. Cho SH, Byun CS, Kim KW, Chang BC, Yoo KJ, Lee S. Preoperative indexed left ventricular dimensions to predict early recovery of left ventricular function after aortic valve replacement for chronic aortic regurgitation. Circ J 2010; 74: 2340-2345.

8. Carasso S, Cohen O, Mutlak D, Adler Z, Lessick J, Reisner SA, et al. Differential effects of afterload on left ventricular long- and shortaxis function: Insights from a clinical model of patients with aortic valve stenosis undergoing aortic valve replacement. Am Heart J 2009; 158: $540-545$.

9. Carasso S, Cohen O, Mutlak D, Adler Z, Lessick J, Aronson D, et al. Relation of myocardial mechanics in severe aortic stenosis to left ventricular ejection fraction and response to aortic valve replacement. Am J Cardiol 2011; 107: $1052-1057$.

10. Ng AC, Delgado V, Bertini M, Antoni ML, van Bommel RJ, van Rijnsoever EP, et al. Alterations in multidirectional myocardial functions in patients with aortic stenosis and preserved ejection fraction: A two-dimensional speckle tracking analysis. Eur Heart J 2011; 32: $1542-1550$.

11. Iwahashi N, Nakatani S, Kanzaki H, Hasegawa T, Abe H, Kitakaze M. Acute improvement in myocardial function assessed by myocardial strain and strain rate after aortic valve replacement for aortic stenosis. J Am Soc Echocardiogr 2006; 19: 1238-1244.

12. Delgado V, Tops LF, van Bommel RJ, van der Kley F, Marsan NA, Klautz RJ, et al. Strain analysis in patients with severe aortic stenosis and preserved left ventricular ejection fraction undergoing surgical valve replacement. Eur Heart J 2009; 30: 3037-3047.

13. Lindqvist P, Bajraktari G, Molle R, Palmerini E, Holmgren A, Mondillo $\mathrm{S}$, et al. Valve replacement for aortic stenosis normalizes subendocardial function in patients with normal ejection fraction. Eur J Echocardiogr 2010; 11: 608-613.

14. Donal E, Bergerot C, Thibault H, Ernande L, Loufoua J, Augeul L, et al. Influence of afterload on left ventricular radial and longitudinal systolic functions: A two-dimensional strain imaging study. Eur J Echocardiogr 2009; 10: 914-921.

15. Devereux RB, Alonso DR, Lutas EM, Gottlieb GJ, Campo E, Sachs I, et al. Echocardiographic assessment of left ventricular hypertrophy: Comparison to necropsy findings. Am J Cardiol 1986; 57: 450458.

16. Lang RM, Bierig M, Devereux RB, Flachskampf FA, Foster E, Pellikka PA, et al. Recommendations for chamber quantification: A report from the American Society of Echocardiography's Guidelines and Standards Committee and the Chamber Quantification Writing Group, developed in conjunction with the European Association of Echocardiography, a branch of the European Society of Cardiology. J Am Soc Echocardiogr 2005; 18: 1440-1463. 
17. Briand M, Dumesnil JG, Kadem L, Tongue AG, Rieu R, Garcia D, et al. Reduced systemic arterial compliance impacts significantly on left ventricular afterload and function in aortic stenosis: Implications for diagnosis and treatment. J Am Coll Cardiol 2005; 46: 291-298.

18. Reichek N, Wilson J, St John Sutton M, Plappert TA, Goldberg S, Hirshfeld JW. Noninvasive determination of left ventricular end-systolic stress: Validation of the method and initial application. Circulation 1982; 65: 99-108.

19. Rosenhek R, Binder T, Porenta G, Lang I, Christ G, Schemper M, et al. Predictors of outcome in severe, asymptomatic aortic stenosis. $N$ Engl J Med 2000; 343: 611-617.

20. Tanaka H, Nesser HJ, Buck T, Oyenuga O, Janosi RA, Winter S, et al. Dyssynchrony by speckle-tracking echocardiography and response to cardiac resynchronization therapy: Results of the speckle tracking and resynchronization (star) study. Eur Heart J 2010; 31: 16901700 .

21. Weidemann F, Herrmann S, Stork S, Niemann M, Frantz S, Lange $\mathrm{V}$, et al. Impact of myocardial fibrosis in patients with symptomatic severe aortic stenosis. Circulation 2009; 120: 577-584.

22. Heymans S, Schroen B, Vermeersch P, Milting H, Gao F, Kassner A, et al. Increased cardiac expression of tissue inhibitor of metalloproteinase- 1 and tissue inhibitor of metalloproteinase- 2 is related to cardiac fibrosis and dysfunction in the chronic pressure-overloaded human heart. Circulation 2005; 112: 1136-1144.
23. Garcia D, Camici PG, Durand LG, Rajappan K, Gaillard E, Rimoldi OE, et al. Impairment of coronary flow reserve in aortic stenosis. $J$ Appl Physiol 2009; 106: 113-121.

24. Abraham WT, Fisher WG, Smith AL, Delurgio DB, Leon AR, Loh $\mathrm{E}$, et al. Cardiac resynchronization in chronic heart failure. $N$ Engl $J$ Med 2002; 346: 1845-1853.

25. St John Sutton MG, Plappert T, Abraham WT, Smith AL, DeLurgio DB, Leon AR, et al. Effect of cardiac resynchronization therapy on left ventricular size and function in chronic heart failure. Circulation 2003; 107: 1985-1990.

26. Suffoletto MS, Dohi K, Cannesson M, Saba S, Gorcsan J 3rd. Novel speckle-tracking radial strain from routine black-and-white echocardiographic images to quantify dyssynchrony and predict response to cardiac resynchronization therapy. Circulation 2006; 113: 960-968.

27. Tanaka H, Hara H, Saba S, Gorcsan J 3rd. Usefulness of threedimensional speckle tracking strain to quantify dyssynchrony and the site of latest mechanical activation. Am J Cardiol 2010; 105: 235242.

28. Tanaka H, Hara H, Adelstein EC, Schwartzman D, Saba S, Gorcsan $\mathrm{J}$ 3rd. Comparative mechanical activation mapping of RV pacing to LBBB by $2 \mathrm{D}$ and $3 \mathrm{D}$ speckle tracking and association with response to resynchronization therapy. JACC Cardiovasc Imaging 2010; 3: $461-471$. 